Discrete Comput Geom 29:485-504 (2003)

DOI: $10.1007 /$ s00454-003-0782-y

\title{
The Representation of Polyhedra by Polynomial Inequalities
}

\author{
Martin Grötschel ${ }^{1}$ and Martin Henk ${ }^{2 *}$ \\ ${ }^{1}$ Konrad-Zuse-Zentrum für Informationstechnik (ZIB), \\ Takustr. 7, D-14195 Berlin-Dahlem, Germany \\ groetschel@zib.de \\ ${ }^{2}$ Abteilung für Analysis, Technische Universität Wien, \\ Wiedner Hauptstr. 8-10/1142, A-1040 Wien, Austria \\ henk@tuwien.ac.at
}

\begin{abstract}
A beautiful result of Bröcker and Scheiderer on the stability index of basic closed semi-algebraic sets implies, as a very special case, that every $d$-dimensional polyhedron admits a representation as the set of solutions of at most $d(d+1) / 2$ polynomial inequalities. Even in this polyhedral case, however, no constructive proof is known, even if the quadratic upper bound is replaced by any bound depending only on the dimension.

Here we give, for simple polytopes, an explicit construction of polynomials describing such a polytope. The number of used polynomials is exponential in the dimension, but in the two- and three-dimensional case we get the expected number $d(d+1) / 2$.
\end{abstract}

\section{Introduction}

By a surprising and striking result of Bröcker and Scheiderer (see [Sc], [Br], [BCR], and [Ma]) every basic closed semi-algebraic set of the form

$$
\mathcal{S}=\left\{x \in \mathbb{R}^{d}: \mathfrak{f}_{1}(x) \geq 0, \ldots, \mathfrak{f}_{l}(x) \geq 0\right\},
$$

where $\mathfrak{f}_{i} \in \mathbb{R}\left[x_{1}, \ldots, x_{d}\right], 1 \leq i \leq l$, are polynomials, can be represented by at most $d(d+1) / 2$ polynomials, i.e., there exist polynomials $\mathfrak{p}_{1}, \ldots, \mathfrak{p}_{d(d+1) / 2} \in \mathbb{R}\left[x_{1}, \ldots, x_{d}\right]$ such that

$$
\mathcal{S}=\left\{x \in \mathbb{R}^{d}: \mathfrak{p}_{1}(x) \geq 0, \ldots, \mathfrak{p}_{d(d+1) / 2}(x) \geq 0\right\}
$$

\footnotetext{
* Current address: Institut für Mathematik, Sekr. MA 6-2, Technische Universität Berlin, Straße des 17. Juni 136, D-10623 Berlin, Germany.
} 
Moreover, in the case of basic open semi-algebraic sets, i.e., $\geq$ is replaced by strict inequality, one can even bound the maximal number of needed polynomials by the dimension $d$ instead of $d(d+1) / 2$.

No explicit constructions, however, of such systems of polynomials are known, even in the very special case of $d$-dimensional convex polyhedra and even if the quadratic upper bound is replaced by any bound depending only on the dimension. In Example 2.10 of [Br] or in Example 4.7 of [ABR] a description of a regular convex $m$-gon in the plane by two polynomials is given. This result was generalized to arbitrary convex polygons and three polynomial inequalities by vom Hofe [vH]. Bernig [Be] proved that, for $d=2$, every convex polygon can even be represented by two polynomial inequalities. The main purpose of this note is to give some basic properties of polynomials describing polyhedra as well as an explicit construction of (exponentially many) polynomials describing simple $d$-polytopes of any dimension $d$.

In order to state the result we fix some notation. A polyhedron $P \subset \mathbb{R}^{d}$ is the intersection of finitely many closed halfspaces, i.e., we can represent it as

$$
P=\left\{x \in \mathbb{R}^{d}: a^{i} \cdot x \leq b_{i}, 1 \leq i \leq m\right\}
$$

for some $a^{i} \in \mathbb{R}^{d}, b_{i} \in \mathbb{R}$. Here $a \cdot x$ denotes the standard inner product on $\mathbb{R}^{d}$. If $P$ is bounded, then it is called a polytope. In general we are only working with $d$-dimensional polyhedra $P \subset \mathbb{R}^{d}$, and, for short, we denote these polyhedra as $d$-polyhedra. A $d$ polyhedron $P$ is called simple if every $k$-dimensional face, $0 \leq k \leq d-1$, belongs to exactly $d-k$ facets of $P$. In the case of polytopes, this is equivalent to the statements that every vertex lies in precisely $d$ facets, or every vertex figure is a simplex (see p. 65 of [Zi]). Since for unbounded polyhedra the above definition of simple polyhedra is not invariant with respect to projective transformation, we call a polyhedron $P$ a strongly simple polyhedron if it is projectively equivalent to a simple polytope, i.e., for a " $P$ permissible" projective transformation $f: \mathbb{R}^{d} \rightarrow \mathbb{R}^{d}$ the closure of $f(P)$, denoted by $\operatorname{cl}(f(P))$, is a simple polytope. For more information about polyhedra, polytopes, and their combinatorial structure we refer to $[\mathrm{MS}]$ and $[\mathrm{Zi}]$.

For polynomials $\mathfrak{p}_{i} \in \mathbb{R}\left[x_{1}, \ldots, x_{d}\right], 1 \leq i \leq l$, we denote by

$$
\mathcal{P}\left(\mathfrak{p}_{1}, \ldots, \mathfrak{p}_{l}\right):=\left\{x \in \mathbb{R}^{d}: \mathfrak{p}_{1}(x) \geq 0, \ldots, \mathfrak{p}_{l}(x) \geq 0\right\}
$$

the associated closed semi-algebraic set and we define

Definition 1.1. A $\mathcal{P}$-representation of a $d$-polyhedron $P \subset \mathbb{R}^{d}$ consists of $l$ polynomials $\mathfrak{p}_{1}, \ldots, \mathfrak{p}_{l} \in \mathbb{R}\left[x_{1}, \ldots, x_{d}\right]$ such that

$$
P=\mathcal{P}\left(\mathfrak{p}_{1}, \ldots, \mathfrak{p}_{l}\right) .
$$

For $d$-polytopes there are two other well known and important representations (see, e.g., [GK] and [Zi]): The representation of $P$ by $m$ vectors $a^{i} \in \mathbb{R}^{d}$ and scalars $b_{i}$ as given in (1.1) is called the $\mathcal{H}$-representation of $P$. Of course, any $\mathcal{H}$-representation may be regarded as a special $\mathcal{P}$-representation of $P$ with linear polynomials (linear forms). As a dual counterpart we have the $\mathcal{V}$-representation of a $d$-polytope $P$ consisting of $n$ points $v^{i} \in \mathbb{R}^{d}$ such that $P$ is the convex hull of these points, i.e., $P=\operatorname{conv}\left\{v^{1}, \ldots, v^{n}\right\}$. 
Both $\mathcal{V}$ - and $\mathcal{H}$-representations are quite powerful and useful representations of polytopes. They admit the computation of the complete combinatorial structure (face-lattice) of the polytope (see [GK] and [Se]) and linear programming problems can be solved in polynomial time with respect to the input sizes of these representations. Many interesting combinatorial optimization problems, however, cannot be effectively solved via these representations because the size of both representations is exponential in the "natural" input size of the combinatorial problem instances. This holds, e.g., for the polytopes associated with the traveling salesman problem or the max cut problem, see [GLS] for details.

On the other hand, the result of Bröcker and Scheiderer tells us that there always exists a $\mathcal{P}$-representation by polynomially many (with respect to the dimension) polynomials, and, therefore, a representation or "good" approximation of a polytope by few polynomial inequalities could lead to a new approach to "hard" combinatorial optimization problems via nonlinear programming tools. In the last section we discuss this connection in more detail as well as the possible outcomes of such an approach.

For a different approach of approximating "discrete problems" by semi-algebraic sets see $[\mathrm{BV}]$, and for related problems involving polynomials and optimization see, e.g., $[\mathrm{Ba}],[\mathrm{BG}],[\mathrm{La}]$, and the references within.

Unfortunately, at the moment our knowledge about polynomials representing or approximating polytopes is rather limited. For arbitrary polytopes we even do not know how to convert-via an algorithm-an $\mathcal{H}$-representation into a $\mathcal{P}$-representation where the number of polynomials depends only on the dimension. For simple polytopes we have the following result.

Theorem 1.2. Let $P \subset \mathbb{R}^{d}$ be a d-dimensional simple polytope given by an $\mathcal{H}$ representation. Then $\mu(d) \leq d^{d}$ polynomials $\mathfrak{p}_{i} \in \mathbb{R}\left[x_{1}, \ldots, x_{d}\right]$ can be constructed such that

$$
P=\mathcal{P}\left(\mathfrak{p}_{1}, \ldots, \mathfrak{p}_{\mu(d)}\right) .
$$

In particular, we can take $\mu(2)=3$ and $\mu(3)=6$.

Since every two-dimensional polygon is simple, Theorem 1.2 includes the result of vom Hofe $[\mathrm{vH}]$.

It is not hard to see that if a polyhedron is given as the set of solutions of polynomial inequalities, then the sum of the total degrees of these polynomials is at least the number of facets (see Proposition 2.1(i)). In fact, the total degrees of the polynomials used in Theorem 1.2 depend on the number of $k$-faces, $k=0, \ldots, d-1$, of the polytope as well as on some metric properties of the polytope. For upper bounds on the degrees in the general semi-algebraic setting we refer to [BM].

All the polynomials that we use in Theorem 1.2 are of a rather special structure, namely, if $P=\left\{x \in \mathbb{R}^{d}: a^{i} \cdot x \leq b_{i}, 1 \leq i \leq m\right\}$, then they can be expressed as

$$
\sum_{\alpha \in \mathbb{N}^{m}, \alpha \geq 0} c_{\alpha} \prod_{i=1}^{m}\left[b_{i}-a^{i} \cdot x\right]^{\alpha_{i}},
$$

where $c_{\alpha}$ are certain non-negative numbers and, of course, only finitely many of them are positive. One possible explanation for this special type is given by a result of Handelman 
[Han] which says that every polynomial that is strictly positive on a polytope $P$ is of that type. His proof is non-constructive, for a more explicit version see [PR].

It seems to be an interesting question to ask for the minimal number of polynomials needed to describe a $d$-polyhedron or $d$-polytope. Therefore we define

Definition 1.3. For a $d$-polyhedron $P \subset \mathbb{R}^{d}$, let $m_{\mathcal{P}}(P)$ be the minimal number of polynomials needed in a $\mathcal{P}$-representation of $P$ and let

$$
\begin{aligned}
& \bar{m}_{\mathcal{P}}(d):=\max \left\{m_{\mathcal{P}}(P): P \subset \mathbb{R}^{d} \text { is a } d \text {-polyhedron }\right\} \\
& m_{\mathcal{P}}(d):=\max \left\{m_{\mathcal{P}}(P): P \subset \mathbb{R}^{d} \text { is a } d \text {-polytope }\right\}
\end{aligned}
$$

We set $m_{\mathcal{P}}\left(\mathbb{R}^{d}\right)=0$, and for a polyhedron $\tilde{P} \subset \mathbb{R}^{d}$ with $\operatorname{dim}(\tilde{P})<d$ we mean by $m_{\mathcal{P}}(\tilde{P})$ the minimal number of polynomials in $\operatorname{dim}(\tilde{P})$-variables, which are needed in order to describe an embedding of $\tilde{P}$ in $\mathbb{R}^{\operatorname{dim}(\tilde{P})}$.

Observe that $m_{\mathcal{P}}(P)$ is invariant with respect to regular affine transformations of $P$. Moreover, it is easy to see that $m_{\mathcal{P}}(P) \geq d$ for every $d$-polytope $P$ (see Corollary 2.2(i)), and together with the result of Bröcker and Scheiderer we obtain

$$
d \leq m_{\mathcal{P}}(d) \leq \bar{m}_{\mathcal{P}}(d) \leq d(d+1) / 2
$$

In Proposition 2.5 we show $\bar{m}_{\mathcal{P}}(d) \leq m_{\mathcal{P}}(d)+1$. Probably, the truth is $\bar{m}_{\mathcal{P}}(d)=m_{\mathcal{P}}(d)$.

There are some trivial examples of polytopes for which $d$ polynomials are sufficient. For instance, the cube $C^{d}=\left\{x \in \mathbb{R}^{d}:-1 \leq x_{i} \leq 1\right\}$ can be written as $C^{d}=\{x \in$ $\left.\mathbb{R}^{d}:-\left(x_{i}\right)^{2}+1 \geq 0\right\}$. Another example is an arbitrary $d$-simplex $T^{d}$. To see this, we may assume without loss of generality that $T^{d}=\left\{x \in \mathbb{R}^{d}: x_{i} \geq 0, x_{1}+\cdots+x_{d} \leq 1\right\}$. Then it is easy to check that

$$
T^{d}=\left\{x \in \mathbb{R}^{d}: x_{i}\left(1-x_{i}-\cdots-x_{d}\right) \geq 0,1 \leq i \leq d\right\} .
$$

Actually, the given representations of a cube and a simplex are special cases of a general construction of polynomial inequalities for prisms and pyramids (see Proposition 2.3), which in particular imply that every three-dimensional prism or pyramid can be described by three polynomials (see Corollary 2.4). However, we are not aware of a representation of a regular crosspolytope $C_{\star}^{d}=\left\{x \in \mathbb{R}^{d}:\left|x_{1}\right|+\cdots+\left|x_{d}\right| \leq 1\right\}$ by $d$ polynomials or of any constructive good upper bound on $m_{\mathcal{P}}\left(C_{\star}^{d}\right)$.

In this context it seems to be worth mentioning that a classical result of Minkowski [Mi] implies that a polytope can be approximated "arbitrarily closely" by only one polynomial inequality, where, of course, the degree of this polynomial is "arbitrarily large." In Section 2 we construct such a polynomial, which will be used in the scope of the proof of Theorem 1.2. Furthermore, in Section 2 we state some simple and basic properties of polynomials describing a polyhedron. In Section 3 we give the construction of the polynomials used in Theorem 1.2 and the proof of this theorem. A generalization of the theorem to strongly simple polyhedra is given in Section 4 (see Corollary 4.1). Finally, in Section 5 we discuss possible outcomes of research on the $\mathcal{P}$-representation of polyhedra associated with hard combinatorial optimization problems. 


\section{Polynomials and Polyhedra}

Let

$$
P=\left\{x \in \mathbb{R}^{d}: a^{i} \cdot x \leq b_{i}, 1 \leq i \leq m\right\}
$$

be a $d$-dimensional polyhedron. We always assume that the representation (2.1) is irredundant, i.e., $P \cap\left\{x \in \mathbb{R}^{d}: a^{i} \cdot x=b_{i}\right\}$ is a facet of $P, 1 \leq i \leq m$. In particular, we have that $b_{i}=h\left(a^{i}\right)$, where $h(\cdot)$ denotes the support function of $P$, i.e.,

$$
h(u)=\sup \{u \cdot x: x \in P\} .
$$

For a non-negative linear combination of vectors $\sum_{i} \rho_{i} u^{i}, \rho_{i} \in \mathbb{R}_{\geq 0}$, we have $h\left(\sum_{i} \rho_{i} u^{i}\right)$ $\leq \sum_{i} \rho_{i} h\left(u^{i}\right)$. The next proposition collects some simple properties of polynomials describing polyhedra.

Proposition 2.1. Let $P=\left\{x \in \mathbb{R}^{d}: a^{i} \cdot x \leq b_{i}, 1 \leq i \leq m\right\}$ be a d-polyhedron and let $\mathfrak{p}_{1}, \ldots, \mathfrak{p}_{l} \in \mathbb{R}\left[x_{1}, \ldots, x_{d}\right]$ such that $P=\mathcal{P}\left(\mathfrak{p}_{1}, \ldots, \mathfrak{p}_{l}\right)$.

(i) Each facet defining linear polynomial $b_{i}-a^{i} \cdot x, i \in\{1, \ldots, m\}$, is a factor of one of the $\mathfrak{p}_{j}$.

(ii) Let $F$ be a $k$-dimensional face of $P$. Then there exist $d-k$ polynomials $\mathfrak{p}_{j_{1}}, \ldots$, $\mathfrak{p}_{j_{d-k}}$, say, such that these polynomials vanish on the affine hull of $F$, i.e.,

$$
\text { aff } F \subset\left\{x \in \mathbb{R}^{d}: \mathfrak{p}_{j_{1}}(x)=\cdots=\mathfrak{p}_{j_{d-k}}(x)=0\right\} .
$$

Proof. (i) Let $F_{i}=P \cap\left\{x \in \mathbb{R}^{d}: a^{i} \cdot x=b_{i}\right\}$. By assumption, $F_{i}$ is a facet of $P$. First we note that for each $y \in F_{i}$ one of the polynomials $\mathfrak{p}_{j}$ has to vanish. Otherwise, if $\mathfrak{p}_{j}(y)>0$ for all $1 \leq j \leq l$ we get by the continuity of polynomials that we can move $y$ in any direction without leaving $P$, which contradicts the property that $y$ belongs to the boundary. Thus we know that the polynomial

$$
\mathfrak{f}(x)=\prod_{j=1}^{l} \mathfrak{p}_{j}(x)
$$

vanishes on $F_{i}$. Hence it vanishes on every segment joining two points of $F_{i}$ and, therefore, it has to be 0 on $\operatorname{aff}\left(F_{i}\right)=\left\{x \in \mathbb{R}^{d}: b_{i}-a^{i} \cdot x=0\right\}$. Thus $b_{i}-a^{i} \cdot x$ is a factor of $\mathfrak{f}(x)$. Furthermore, since $b_{i}-a^{i} \cdot x$ is irreducible, it has to be a factor of one of the $\mathfrak{p}_{j}$ (see, e.g., p. 148 of [CLO]).

(ii) We use induction with respect to the dimension $k$ of the face $F$ and we start with $k=d-1$. In this case the statement follows immediately from (i). So let $k<d-1$ and let $G$ be a $(k+1)$-face containing $F$. By induction we can assume that $\operatorname{aff}(G) \subset\{x \in$ $\left.\mathbb{R}^{d}: \mathfrak{p}_{1}(x)=\mathfrak{p}_{2}(x)=\cdots=\mathfrak{p}_{d-k-1}(x)=0\right\}$ and so

$$
G=\operatorname{aff}(G) \cap P=\left\{x \in \operatorname{aff}(G): \mathfrak{p}_{d-k}(x) \geq 0, \ldots, \mathfrak{p}_{l}(x) \geq 0\right\} .
$$

With respect to the $(k+1)$-dimensional polytope $G$ in the space $\operatorname{aff}(G)$ the face $F$ is a facet and so we can conclude that one of the polynomials $\mathfrak{p}_{d-k}, \ldots, \mathfrak{p}_{l}$ vanishes on $\operatorname{aff}(F)$. 
As an immediate consequence of Proposition 2.1(ii) we note

\section{Corollary 2.2.}

(i) Let $F$ be a non-empty face of a d-polyhedron $P \subset \mathbb{R}^{d}$. Then

$$
m_{\mathcal{P}}(P) \geq m_{\mathcal{P}}(F)+d-\operatorname{dim}(F) .
$$

In particular, $m_{\mathcal{P}}(P) \geq d$ for $d$-polytopes.

(ii) $m_{\mathcal{P}}(d+1) \geq m_{\mathcal{P}}(d)+1$ and $\bar{m}_{\mathcal{P}}(d+1) \geq \bar{m}_{\mathcal{P}}(d)+1$.

Proof. Let $P=\mathcal{P}\left(\mathfrak{p}_{1}, \ldots, \mathfrak{p}_{l}\right)$. By Proposition 2.1(ii) we may assume that $\operatorname{aff}(F) \subset$ $\left\{x \in \mathbb{R}^{d}: \mathfrak{p}_{1}(x)=\cdots=\mathfrak{p}_{d-\operatorname{dim}(F)}(x)=0\right\}$. Thus

$$
F=\operatorname{aff}(F) \cap P=\left\{x \in \operatorname{aff}(F): \mathfrak{p}_{d-\operatorname{dim}(F)+1}(x) \geq 0, \ldots, \mathfrak{p}_{l}(x) \geq 0\right\}
$$

and so $m_{\mathcal{P}}(F) \leq m_{\mathcal{P}}(P)-(d-\operatorname{dim}(F))$. If $P$ is a polytope, then we may choose for $F$ a vertex and get $m_{\mathcal{P}}(P) \geq d$.

For (ii) let $Q$ be a $d$-polytope with $m_{\mathcal{P}}(Q)=m_{\mathcal{P}}(d)$. Now we take any $(d+1)$ polytope that has $Q$ as a facet and then we can conclude from (i) that $m_{\mathcal{P}}(P) \geq m_{\mathcal{P}}(d)+1$. Of course, the polyhedral case can be treated analogously.

The next statement gives some information on $m_{\mathcal{P}}(P)$ for $d$-prisms and $d$-pyramids. A $d$-polytope $P$ is called a $d$-pyramid ( $d$-prism) with basis $Q$, where $Q$ is a $(d-1)$ polytope, if there exists a $v \in \mathbb{R}^{d}$ such that $P=\operatorname{conv}\{Q, v\}(P=Q+\operatorname{conv}\{0, v\}=$ $\{q+\lambda v: q \in Q, 0 \leq \lambda \leq 1\})$.

Proposition 2.3. Let $P$ be a d-prism or a d-pyramid with basis $Q$. Then

$$
m_{\mathcal{P}}(P)=m_{\mathcal{P}}(Q)+1
$$

Proof. Since in both cases the $(d-1)$-polytope $Q$ is a facet of $P$ we get from Corollary 2.2(i)

$$
m_{\mathcal{P}}(P) \geq m_{\mathcal{P}}(Q)+1
$$

In order to show the reverse inequality we start with a $d$-dimensional pyramid $P=$ $\operatorname{conv}\{Q, v\}$ and without loss of generality we assume that

$$
\text { (i) } Q \subset\left\{x \in \mathbb{R}^{d}: x_{d}=0 \text { and } \sum_{i=1}^{d-1}\left(x_{i}\right)^{2} \leq 1\right\}, \quad \text { (ii) } v=(0, \ldots, 0,1)^{\top} \text {. }
$$

Let $l=m_{\mathcal{P}}(Q)$ and $\mathfrak{p}_{1}, \ldots, \mathfrak{p}_{l} \in \mathbb{R}\left[x_{1}, \ldots, x_{d-1}\right]$ such that $Q=\mathcal{P}\left(\mathfrak{p}_{1}, \ldots, \mathfrak{p}_{l}\right)$. Furthermore, we denote by $p$ the maximum of the total degrees of the polynomials $\mathfrak{p}_{j}$, $1 \leq j \leq l$, and let

$$
\begin{aligned}
\tilde{\mathfrak{p}}_{j}(x) & =\left(1-x_{d}\right)^{p} \cdot \mathfrak{p}_{j}\left(\frac{x_{1}}{1-x_{d}}, \ldots, \frac{x_{d-1}}{1-x_{d}}\right), \quad 1 \leq j \leq l, \\
\mathfrak{p}(x) & =x_{d}\left(1-x_{d}-x_{d}\left(\left(x_{1}\right)^{2}+\cdots+\left(x_{d-1}\right)^{2}\right)\right)
\end{aligned}
$$


Observe that $\tilde{\mathfrak{p}}_{j}(x) \in \mathbb{R}\left[x_{1}, \ldots, x_{d}\right], 1 \leq j \leq l$. Next we claim that

$$
P=\mathcal{P}\left(\tilde{\mathfrak{p}}_{1}, \ldots, \tilde{\mathfrak{p}}_{l}, \mathfrak{p}\right) .
$$

To see this we first note that for $0 \leq \lambda \leq 1$,

$$
P \cap\left\{x \in \mathbb{R}^{d}: x_{d}=\lambda\right\}=(1-\lambda) Q+\lambda(0, \ldots, 0,1)^{\top} .
$$

A simple calculation shows that, for $0 \leq \lambda<1$,

$$
\begin{aligned}
P & \cap\left\{x \in \mathbb{R}^{d}: x_{d}=\lambda\right\} \\
& =\left\{\left(x_{1}, \ldots, x_{d-1}, \lambda\right)^{\top} \in \mathbb{R}^{d}: \tilde{\mathfrak{p}}_{j}\left(x_{1}, \ldots, x_{d-1}, \lambda\right) \geq 0,1 \leq j \leq l\right\} .
\end{aligned}
$$

Next we observe that, for $x \in P$, we have $0 \leq x_{d} \leq 1$. By (2.6) and assumption (2.3(i)) we conclude that

$$
\left(x_{1}\right)^{2}+\cdots+\left(x_{d-1}\right)^{2} \leq\left(1-x_{d}\right)^{2} \quad \text { for all } \quad x \in P .
$$

Hence $\mathfrak{p}(x) \geq 0$ for $x \in P$ and together with (2.7) we get $P \backslash\left\{(0, \ldots, 0,1)^{\top}\right\} \subset$ $\mathcal{P}\left(\tilde{\mathfrak{p}}_{1}, \ldots, \tilde{\mathfrak{p}}_{l}, \mathfrak{p}\right)$ and consequently $P \subset \mathcal{P}\left(\tilde{\mathfrak{p}}_{1}, \ldots, \tilde{\mathfrak{p}}_{l}, \mathfrak{p}\right)$.

For the reverse inclusion we notice that $\mathfrak{p}(x) \geq 0$ implies $0 \leq x_{d} \leq 1$ and with (2.7) we obtain

$$
\mathcal{P}\left(\tilde{\mathfrak{p}}_{1}, \ldots, \tilde{\mathfrak{p}}_{l}, \mathfrak{p}\right) \backslash\left\{x \in \mathbb{R}^{d}: x_{d}=1\right\} \subset P .
$$

Since for $x_{d}=1$ the inequality $\mathfrak{p}(x) \geq 0$ becomes $\left(x_{1}\right)^{2}+\cdots+\left(x_{d-1}\right)^{2} \leq 0$ we conclude that

$$
\mathcal{P}\left(\tilde{\mathfrak{p}}_{1}, \ldots, \tilde{\mathfrak{p}}_{l}, \mathfrak{p}\right) \cap\left\{x \in \mathbb{R}^{d}: x_{d}=1\right\}=\left\{(0, \ldots, 0,1)^{\top}\right\} .
$$

Hence we also have $\mathcal{P}\left(\tilde{\mathfrak{p}}_{1}, \ldots, \tilde{\mathfrak{p}}_{l}, \mathfrak{p}\right) \subset P$. Thus $(2.5)$ is shown and so we have $m_{\mathcal{P}}(P) \leq$ $m_{\mathcal{P}}(Q)+1$. Together with (2.2) the statement of the proposition is verified for pyramids.

If $P=Q+\operatorname{conv}\{0, v\}$ is a $d$-prism over the basis $Q$ and if we assume again that $v=(0, \ldots, 0,1)^{\top}, Q \subset\left\{x \in \mathbb{R}^{d}: x_{d}=0\right\}$, and $Q=\mathcal{P}\left(\mathfrak{p}_{1}, \ldots, \mathfrak{p}_{m_{\mathcal{P}}(Q)}\right)$, then it is easy to check that

$$
P=\left\{x \in \mathbb{R}^{d}: \mathfrak{p}_{j}\left(x_{1}, \ldots, x_{d-1}\right) \geq 0,1 \leq j \leq m_{\mathcal{P}}(Q), x_{d}\left(1-x_{d}\right) \geq 0\right\}
$$

Since every two-dimensional polygon can be described by two polynomials (see [Be]), Proposition 2.3 gives

Corollary 2.4. Let $P$ be a three-dimensional prism or pyramid. Then

$$
m_{\mathcal{P}}(P)=3 \text {. }
$$

Next we study the relation between $m_{\mathcal{P}}(d)$ and $\bar{m}_{\mathcal{P}}(d)$. Obviously, we have $m_{\mathcal{P}}(d) \leq$ $\bar{m}_{\mathcal{P}}(d)$. In order to bound $\bar{m}_{\mathcal{P}}(d)$ in terms of $m_{\mathcal{P}}(d)$, we apply a standard technique from Discrete Geometry, which "makes an unbounded pointed polyhedron bounded," namely, projective transformations. 
Proposition 2.5. Let $d \geq 2$. Then

$$
m_{\mathcal{P}}(d) \leq \bar{m}_{\mathcal{P}}(d) \leq m_{\mathcal{P}}(d)+1 .
$$

Proof. In order to prove the upper bound on $\bar{m}_{\mathcal{P}}(d)$ let $P$ be a $d$-polyhedron such that $\bar{m}_{\mathcal{P}}(d)=m_{\mathcal{P}}(P)$. Let $G$ be a non-empty face of minimal dimension of $P$, and we assume that $0 \in G$. Suppose that $\operatorname{dim}(G)>0$. Then the intersection of $P$ with the orthogonal complement of $\operatorname{lin}(G)$, the linear hull of $G$, is a lower-dimensional polyhedron $Q$, say. Since $P=Q+\operatorname{lin}(G)=\{q+g: q \in Q, g \in \operatorname{lin}(G)\}$ any $\mathcal{P}$-representation of $Q$ can easily be converted to a $\mathcal{P}$-representation of $P$ with the same number of polynomials. With the help of Corollary 2.2(ii) we get the contradiction

$$
\bar{m}_{\mathcal{P}}(d)=m_{\mathcal{P}}(P)=m_{\mathcal{P}}(Q) \leq \bar{m}_{\mathcal{P}}(\operatorname{dim}(Q))<\bar{m}_{\mathcal{P}}(d) .
$$

Therefore, we can assume that the origin is a vertex of $P$. Thus, we can find a vector $c \in \mathbb{R}^{d}$ with $c \cdot x>0$ for all $x \in P \backslash\{0\}$. Let $f: \mathbb{R}^{d} \rightarrow \mathbb{R}^{d}$ be the projective map

$$
f(x)=\frac{x}{c \cdot x+1} .
$$

Then we can describe $f(P)$ by a set of inequalities of the form $f(P)=\left\{x \in \mathbb{R}^{d}: A x \leq\right.$ $b, c \cdot x<1\}$, for a certain matrix $A \in \mathbb{R}^{m \times d}$ and a vector $b \in \mathbb{R}^{m}$. The inequality $c \cdot x<1$ corresponds to the points at infinity.

We conclude that the set (the closure of $f(P)$ )

$$
\operatorname{cl}(f(P))=\left\{x \in \mathbb{R}^{d}: A x \leq b, c \cdot x \leq 1\right\}
$$

is a $d$-dimensional polytope. Hence we get can find a $\mathcal{P}$-representation of $\operatorname{cl}(f(P))$ by polynomials $\mathfrak{p}_{i}, i \in I$, say, with $\# I \leq m_{\mathcal{P}}(d)$. So we may write

$$
f(P)=\left\{x \in \mathbb{R}^{d}: \mathfrak{p}_{i}(x) \geq 0, i \in I, c \cdot x<1\right\} .
$$

Thus

$$
P=\left\{x \in \mathbb{R}^{d} \backslash\left\{x \in \mathbb{R}^{d}: c \cdot x=-1\right\}: \mathfrak{p}_{i}(f(x)) \geq 0, i \in I, c \cdot f(x)<1\right\} .
$$

Since $c \cdot f(x)<1$ is equivalent to $c \cdot x+1>0$ we may multiply all rational functions $\mathfrak{p}_{i}(f(x))$ by suitable powers of $c \cdot x+1$ and obtain some polynomials $\tilde{\mathfrak{p}}_{i}(x)$, say, such that

$$
P=\left\{x \in \mathbb{R}^{d}: \tilde{\mathfrak{p}}_{i}(x) \geq 0, i \in I, c \cdot x+1>0\right\} .
$$

Since $c \cdot x \geq 0$ for all $x \in P$ we may replace the last inequality in this representation by $c \cdot x \geq 0$ and since $\# I \leq m_{\mathcal{P}}(d)$ the proposition is shown.

In the next lemma a strictly convex polynomial $\mathfrak{p}$ is constructed such that the convex body $K=\left\{x \in \mathbb{R}^{d}: \mathfrak{p}(x) \leq 1\right\}$ is not "too far away" from $P$. Here the distance between convex bodies $K_{1}, K_{2}$ will be measured by the Hausdorff distance $\operatorname{dist}\left(K_{1}, K_{2}\right)$, i.e.,

$$
\operatorname{dist}\left(K_{1}, K_{2}\right):=\max \left\{\max _{x \in K_{1}} \min _{y \in K_{2}}\|x-y\|, \max _{x \in K_{2}} \min _{y \in K_{1}}\|x-y\|\right\},
$$


where $\|\cdot\|$ denotes the Euclidean norm. Furthermore, for a bounded set $S \subset \mathbb{R}^{d}$, the diameter is denoted by $\operatorname{diam}(S)$, i.e.,

$$
\operatorname{diam}(S):=\max \{\|x-y\|: x, y \in S\} .
$$

In order to construct this strictly convex polynomial we follow an approach of Hammer [Ham], but since we need a slightly different approximation we give the short proof. For similar results see [Fi] and [We].

Lemma 2.6. Let $P=\left\{x \in \mathbb{R}^{d}: a^{i} \cdot x \leq b_{i}, 1 \leq i \leq m\right\}$ be a d-dimensional polytope. For $1 \leq i \leq m$ let

$$
\mathfrak{v}_{i}(x):=\frac{2 a^{i} \cdot x-h\left(a^{i}\right)+h\left(-a^{i}\right)}{h\left(a^{i}\right)+h\left(-a^{i}\right)} .
$$

Let $\varepsilon>0, p>\ln (m) /(2 \ln (1+2 \varepsilon /((d+1) \operatorname{diam}(P))))$,

$$
\mathfrak{p}_{\varepsilon}(x):=\sum_{i=1}^{m} \frac{1}{m}\left[\mathfrak{y}_{i}(x)\right]^{2 p} \quad \text { and } \quad K_{\varepsilon}:=\left\{x \in \mathbb{R}^{d}: \mathfrak{p}_{\varepsilon}(x) \leq 1\right\} .
$$

Then we have $P \subset K_{\varepsilon}$ and $\operatorname{dist}\left(P, K_{\varepsilon}\right) \leq \varepsilon$.

Proof. Since $\left|\mathfrak{v}_{i}(x)\right| \leq 1$ for all $x \in P$ we certainly have $P \subset K_{\varepsilon}$. Without loss of generality let the origin be the center of gravity of $P$ and let $\lambda=\varepsilon / \operatorname{diam}(P)$. First we check that $K_{\varepsilon} \subset P_{\lambda}=\left\{x \in \mathbb{R}^{d}: a^{i} \cdot x \leq(1+\lambda) b_{i}, 1 \leq i \leq m\right\}$. Let $y \notin P_{\lambda}$. Then we may assume $a^{1} \cdot y>(1+\lambda) h\left(a^{1}\right)$ which implies

$$
\mathfrak{v}_{1}(y)>1+2 \lambda \frac{h\left(a^{1}\right)}{h\left(a^{1}\right)+h\left(-a^{1}\right)} \geq 1+\frac{2 \lambda}{d+1},
$$

where the last inequality follows from the choice of the origin as the center of gravity (see p. 52 of $[\mathrm{BF}]$ ). By the lower bound on $p$ we conclude $(1 / m) \mathfrak{v}_{1}(y)^{2 p}>1$ and thus $\mathfrak{p}_{\varepsilon}(y)>1$, which shows $y \notin K_{\varepsilon}$. Finally we observe that $\operatorname{dist}\left(P, P_{\lambda}\right) \leq \lambda$ $\operatorname{diam}(P)=\varepsilon$.

\section{Proof of Theorem 1.2}

In the following let

$$
P=\left\{x \in \mathbb{R}^{d}: a^{i} \cdot x \leq b_{i}, 1 \leq i \leq m\right\}
$$

be a convex $d$-dimensional simple polytope with $m$ facets. We further assume that we know all $k$-faces of the polytope as well as the facets containing a given face. This information can be obtained from the $\mathcal{H}$-representation above by several (exponential time and space) algorithms (see [Se]).

We remark that every $k$-face of $P$ is contained in exactly $d-k$ facets. The set of all $k$-dimensional faces is denoted by $\mathcal{F}_{k}, 0 \leq k \leq d-1$. For a $k$-face $F$ of $P$, let 
$[F]_{1}, \ldots,[F]_{d-k},[F]_{1} \leq \cdots \leq[F]_{d-k}$, be all indices of vectors $a^{i}$ such that $a^{i} \cdot x=b_{i}$, for all $x \in \operatorname{aff}(F)$. In other words, these are the ordered indices of all facets containing $F$.

Next, for a $k$-face $F$ and a positive integral vector $w \in \mathbb{N}^{d-k}$, we define

$$
a(F, w):=\sum_{j=1}^{d-k} w_{j} a^{[F]_{j}} .
$$

Observe that $a(F, w)$ is a support vector of $F$, i.e.,

$$
F=P \cap\left\{x \in \mathbb{R}^{d}: a(F, w) \cdot x=h(a(F, w))\right\} .
$$

Moreover, since $F$ is contained in all the facets corresponding to the vectors $a^{[F]_{j}}$ we note that

$$
h(a(F, w))=\sum_{j=1}^{d-k} w_{j} h\left(a^{[F]_{j}}\right) .
$$

With $w \in \mathbb{N}^{d-k}$ and the set $\mathcal{F}_{k}$ of all $k$-faces we associate the polynomial

$$
\mathfrak{p}_{k, w}(x):=\prod_{F \in \mathcal{F}_{k}}[h(a(F, w))-a(F, w) \cdot x] .
$$

So, for a fixed $w$ the polynomial $\mathfrak{p}_{k, w}(x)$ is the product of all those supporting hyperplanes of all $k$-faces of $P$ which can be written as in (3.1). Since we are only interested in finitely many different support vectors of the type $a(F, w)$ at a given face $F$ we define certain sets of integral vectors:

$$
\mathcal{W}_{d-1}:=\{(1)\}, \quad \mathcal{W}_{d-k}:=\left\{\left(2^{l_{1}}, \ldots, 2^{l_{k}}\right)^{\top}: 0 \leq l_{i} \leq k-2\right\}, \quad 2 \leq k \leq d .
$$

In particular we have $\mathcal{W}_{d-2}=\{(1,1)\}$ and

$$
\# \mathcal{W}_{k}=(d-k-1)^{d-k} \text {. }
$$

The meaning of these sets $\mathcal{W}_{k}$ is explained in the next lemma.

Lemma 3.1. Let $P$ be a simple d-dimensional polytope. Let $k \geq 1, F, G \in \mathcal{F}_{k}$ with $F \cap G \neq \emptyset$, and let $w \in \mathcal{W}_{k}, y \in \mathbb{R}^{d}$ such that

$$
h(a(F, w))-a(F, w) \cdot y<0 \quad \text { and } \quad h(a(G, w))-a(G, w) \cdot y \leq 0 .
$$

Then there exists a $\tilde{w} \in \mathcal{W}_{\operatorname{dim}(F \cap G)}$ such that

$$
h(a(F \cap G, \tilde{w}))-a(F \cap G, \tilde{w}) \cdot y<0 .
$$

Proof. In view of (3.2) we conclude from (3.5) that

$$
\begin{aligned}
& \sum_{j=1}^{d-k} w_{j}\left(h\left(a^{[F]_{j}}\right)-a^{[F]_{j}} \cdot y\right)<0, \\
& \sum_{j=1}^{d-k} w_{j}\left(h\left(a^{[G]_{j}}\right)-a^{[G]_{j}} \cdot y\right) \leq 0 .
\end{aligned}
$$


Let $I_{1}=\left\{[F]_{1}, \ldots,[F]_{d-k}\right\}, I_{2}=\left\{[G]_{j}: h\left(a^{[G]_{j}}\right)-a^{[G]_{j}} \cdot y \leq 0\right\}, I_{3}=\left\{[G]_{j}: h\left(a^{[G]_{j}}\right)-\right.$ $\left.a^{[G]_{j}} \cdot y>0\right\}$, and let $I=I_{1} \cup I_{2} \cup I_{3}$.

Further, we need a map $\tau$ that gives, for a $k$-face $F$ and a number $q \in\left\{[F]_{1}, \ldots\right.$, $\left.[F]_{d-k}\right\}$, the position of $q$ with respect to the ordered list $[F]_{1}, \ldots,[F]_{d-k}$. In particular we have $\tau\left(F,[F]_{j}\right)=j$. Now we merge the two normal vectors $a(F, w), a(G, w)$ in the following way: Let

$$
\begin{aligned}
a= & \sum_{j \in I_{1} \cap I_{2}} 2 \max \left\{w_{\tau(F, j)}, w_{\tau(G, j)}\right\} a^{j}+\sum_{j \in I_{1} \cap I_{3}} a^{j} \\
& +\sum_{j \in\left(I_{2} \cup I_{3}\right) \backslash I_{1}} w_{\tau(G, j)} a^{j}+\sum_{j \in I_{1} \backslash\left(I_{2} \cup I_{3}\right)} w_{\tau(F, j)} a^{j} .
\end{aligned}
$$

On account of (3.6) we have

$$
h(a)-a \cdot y<0 .
$$

Since the polytope is simple, the assumption $F \cap G \neq \emptyset$ implies that the vectors $\left\{a^{j}: j \in\right.$ $I\}$ are the vectors of all facets containing the face $F \cap G$ and by construction we may write $a=\sum_{j \in I} \tilde{w}_{i_{j}} a^{j}$ for some numbers $\tilde{w}_{i_{j}}=2^{l_{i j}}$ with $0 \leq l_{i_{j}} \leq d-k-1$.

Thus we have $a=a(F \cap G, \tilde{w})$ for a certain vector $\tilde{w} \in \mathcal{W}_{\operatorname{dim}(F \cap G)}$.

We note that from the proof of Lemma 3.1 it follows that it suffices to define the set $\mathcal{W}_{d-3}$ as

$$
\mathcal{W}_{d-3}=\left\{(1,1,2)^{\top},(1,2,1)^{\top},(2,1,1)^{\top}\right\}
$$

Lemma 3.1 says that if two linear factors of a polynomial $\mathfrak{p}_{k, w}, k \in\{1, \ldots, d-1\}$, $w \in \mathcal{W}_{k}$, are non-positive and at least one is negative, then there exists a linear factor of a polynomial of the type $\mathfrak{p}_{\tilde{k}, \tilde{w}}, \tilde{k}<k, \tilde{w} \in \mathcal{W}_{\tilde{k}}$, which has to be negative, too. Therefore, with these sets $\mathcal{W}_{k}$ we associate the following sets of polynomials:

$$
\mathfrak{P}_{k}=\left\{\mathfrak{p}_{k, w}(x): w \in \mathcal{W}_{k}\right\}, \quad k=0, \ldots, d-1 .
$$

$\mathfrak{P}_{d-1}, \mathfrak{P}_{d-2}$ consist of only one polynomial, $\# \mathfrak{P}_{d-3}=3$ and for $0 \leq k \leq d-3$ we have (see (3.4))

$$
\# \mathfrak{P}_{k}=(d-k-1)^{d-k} .
$$

We need one more polynomial. To this end we set, for two vectors $a, b \in \mathbb{R}^{d} \backslash\{0\}$,

$$
U(a, b)=\left\{x \in \mathbb{R}^{d}: a \cdot x \geq h(a) \text { and } b \cdot x \geq h(b)\right\} .
$$

$U(a, b)$ is a closed set and so we can define

$$
\varepsilon(a, b)=\min \{\|x-y\|: x \in P, y \in U(a, b)\} .
$$

Since $P \subset\left\{x \in \mathbb{R}^{d}: a \cdot x \leq h(a), b \cdot x \leq h(b)\right\}$ and both planes $\left\{x \in \mathbb{R}^{d}: a \cdot x=h(a)\right\}$, $\left\{x \in \mathbb{R}^{d}: b \cdot x=h(b)\right\}$ are supporting hyperplanes we have

$$
\varepsilon(a, b)=0 \quad \Longleftrightarrow \quad\left\{x \in \mathbb{R}^{d}: a \cdot x=h(a) \text { and } b \cdot x=h(b)\right\} \cap \mathcal{F}_{0} \neq \emptyset .
$$


For $0 \leq k \leq d-1$ we set

$$
\varepsilon_{k}=\min \left\{\varepsilon(a(F, w), a(\tilde{F}, w))>0: F \neq \tilde{F} \in \mathcal{F}_{k}, w \in \mathcal{W}_{k}\right\} .
$$

We note that for different vertices $v, \tilde{v} \in \mathcal{F}_{0}, w \in \mathcal{W}_{0}$, we always have (see (3.10))

$$
\varepsilon(a(v, w), a(\tilde{v}, w))>0 .
$$

Finally let $\bar{\varepsilon}$ satisfy

$$
0<\bar{\varepsilon}<\min \left\{\varepsilon_{k}: 0 \leq k \leq d-1\right\} .
$$

With respect to $\bar{\varepsilon}$ let $\mathfrak{p}_{\bar{\varepsilon}}(x)$ be the polynomial according to Lemma 2.6 and let

$$
\begin{aligned}
& \mathcal{V}_{1}(P)=\left\{x \in \mathbb{R}^{d}: \mathfrak{P}_{k}(x) \geq 0,1 \leq k \leq d-1, \mathfrak{p}_{\bar{\varepsilon}}(x) \leq 1\right\}, \\
& \mathcal{V}_{2}(P)=\left\{x \in \mathbb{R}^{d}: \mathfrak{P}_{0}(x) \geq 0\right\} .
\end{aligned}
$$

Here for a set of polynomials $\mathfrak{P}$, say, $\mathfrak{P}(x) \geq 0$ means $\mathfrak{p}(x) \geq 0$ for all $\mathfrak{p}(x) \in \mathfrak{P}$.

Before giving the last piece of the proof of Theorem 1.2 we remark that in order to find a number $\bar{\varepsilon}$ satisfying (3.13) we have to calculate several distances $\varepsilon(a, b)$. In general, $\varepsilon(a, b)$ can be calculated (or sufficiently well approximated) by several linearprogramming-based methods (see [MSW]). In particular, depending on the input size of the polytope and the vectors $a, b$ one can give a lower bound on this distance if it is positive. Thus for a given polytope we can calculate such an $\bar{\varepsilon}$ and hence the polynomial $\mathfrak{p}_{\bar{\varepsilon}}(x)$.

Proof of Theorem 1.2. On account of (3.9) and (3.7) the theorem will follow from the identity

$$
P=\mathcal{V}_{1}(P) \cap \mathcal{V}_{2}(P)
$$

Obviously, by the definition of all these polynomials via support vectors and by Lemma 2.6 we know that $P$ is contained in the set on the right-hand side. In order to prove the reverse inclusion we first claim

Claim 3.2. Let $y \notin P$, but $y \in \mathcal{V}_{1}(P)$. Then there exists a vertex $v$ of $P$ and $a w \in \mathcal{W}_{0}$ such that $h(a(v, w))-a(v, w) \cdot y<0$.

Since $y \notin P$ at least one of the inequalities $a^{i} \cdot x \leq b_{i}, i \in\{1, \ldots, m\}$, is violated and so we may define $k$ as the smallest dimension such that there exists a face $F \in \mathcal{F}_{k}$ and a $w \in \mathcal{W}_{k}$ with $h(a(F, w))-a(F, w) \cdot y<0$. Suppose $k>0$. Since $y \in \mathcal{V}_{1}(P)$ we have $p_{k, w}(y) \geq 0$ and so there must exist another $k$-face $G$ with $h(a(G, w))-a(G, w) \cdot y \leq 0$. Hence we have

$$
y \in U(a(F, w), a(G, w)) .
$$

If $\varepsilon(a(F, w), a(G, w))>0$, then we get from the definition of $\bar{\varepsilon}$ and the approximating property of the polynomial $\mathfrak{p}_{\bar{\varepsilon}}(x)$ (see Lemma 2.6) that $\mathfrak{p}_{\bar{\varepsilon}}(y)>1$. Thus we can assume that $\varepsilon(a(F, w), a(G, w))=0$ and from (3.10) we get $F \cap G \neq \emptyset$. Therefore we may apply Lemma 3.1 and we get a contradiction of the minimality of $k$. This shows Claim 3.2. 
Now let $y \notin P$. We want to show that $y$ is not contained in the set $\mathcal{V}_{1}(P) \cap \mathcal{V}_{2}(P)$. Suppose that $y \in \mathcal{V}_{1}(P) \cap \mathcal{V}_{2}(P)$. By Claim 3.2 we may assume that there exists a vertex $v \in \mathcal{F}_{0}$ and a $w \in \mathcal{W}_{0}$ such that $h(a(v, w))-a(v, w) \cdot y<0$. However, $y \in \mathcal{V}_{2}(P)$ implies $\mathfrak{p}_{0, w}(y) \geq 0$ and thus there exists another vertex $\tilde{v}$ with $h(a(\tilde{v}, w))-a(\tilde{v}, w) \cdot y \leq$ 0 . Therefore we have

$$
y \in W(a(v, w), a(\tilde{v}, w)) .
$$

Next we observe that $\varepsilon(a(v, w), a(\tilde{v}, w))>0$ (see (3.12)) and by the definition of $\bar{\varepsilon}$ we conclude $\mathfrak{p}_{\bar{\varepsilon}}(y)>1$, which gives the contradiction $y \notin \mathcal{V}_{1}(P)$.

\section{Remarks}

First we want to generalize Theorem 1.2 to the class of strongly simple polyhedra. As in the case of polytopes, an $\mathcal{H}$-representation of a polyhedron $P$ is a description of $P$ by linear inequalities of the form (1.1).

Corollary 4.1. Let $P \subset \mathbb{R}^{d}$ be ad-dimensional strongly simple polyhedron given by an $\mathcal{H}$-representation. Then $\mu(d) \leq d^{d}$ polynomials $\mathfrak{p}_{i} \in \mathbb{R}\left[x_{1}, \ldots, x_{d}\right]$ can be constructed such that

$$
P=\left\{x \in \mathbb{R}^{d}: \mathfrak{p}_{i}(x) \geq 0, \quad 1 \leq i \leq \mu(d)\right\} .
$$

Proof. The proof is just a combination of the proofs of Proposition 2.5 and Theorem 1.2. As in the proof of Proposition 2.5 we first note that we can assume that $P$ has a vertex. Next we apply a projective transformation $f(x)=x /(c \cdot x+1)$ such that $\operatorname{cl}(f(P))$ becomes a polytope. By the definition of strongly simple polyhedra, $\operatorname{cl}(f(P))$ is a $d$ dimensional simple polytope. Hence, from Theorem 1.2, we get a representation of the type

$$
f(P)=\left\{x \in \mathbb{R}^{d}: \mathfrak{p}_{i}(x) \geq 0, i \in I, c \cdot x<1\right\}
$$

with certain polynomials $\mathfrak{p}_{i}(x), i \in I, \# I \leq \mu(d)$. Now we can proceed as in the proof of Proposition 2.5 in order to get a $\mathcal{P}$-representation of $P$ with $\mu(d)+1$ polynomials. A closer look on the number $\mu(d)$ shows that $\mu(d)<d^{d}$ for $d \geq 2$ (see (3.9)) and so the assertion is proved.

We remark that the proofs of Theorem 1.2 and of Corollary 4.1 can be adapted such that for arbitrary polyhedra a representation by polynomials is obtained where the number of polynomials depends exponentially on the dimension and the maximal degree of a vertex of the polytope. In other words, degeneracy in the sense of linear programming leads to additional difficulties. Since, however, the main problem is to find a representation of a polytope by a few polynomials we omit a proof of this statement.

In the two-dimensional case the meaning of the polynomials $\mathfrak{p}_{1}(x), \mathfrak{p}_{0}(x)$, and $\mathfrak{p}_{\bar{\varepsilon}}(x)$ can be easily illustrated. Suppose the polygon is the 7-gon depicted in these pictures. Then the shaded regions on the left-hand side of Fig. 1 shows all points in the plane 


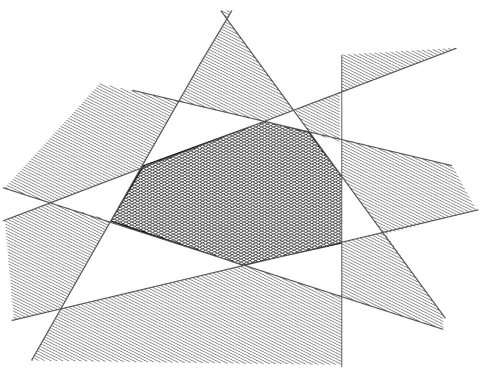

$\left\{x \in \mathbb{R}^{d}: \mathfrak{p}_{1}(x) \geq 0\right\}$

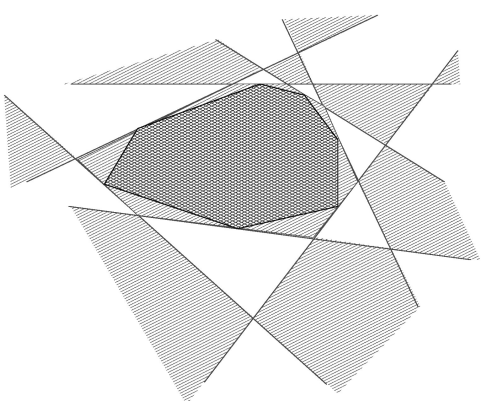

$\left\{x \in \mathbb{R}^{d}: \mathfrak{p}_{0}(x) \geq 0\right\}$

Fig. 1

that satisfy the inequality $\mathfrak{p}_{1}(x) \geq 0$, whereas the shaded regions on the right-hand side correspond to the points $\mathfrak{p}_{0}(x) \geq 0$. If we intersect the shaded regions of both pictures we get the points satisfying both inequalities (see Fig. 2).

We see that all the shaded points that do not belong to the polygon are "far away" from the polygon and thus we can cut them off with the inequality $\mathfrak{p}_{\bar{\varepsilon}}(x) \leq 1$.

Now let $P=\left\{x \in \mathbb{R}^{3}: a^{i} \cdot x \leq b_{i}, 1 \leq i \leq m\right\}$ be a simple three-dimensional convex polytope. With the notation from Section 3 we get the following polynomials (see (3.7)):

$$
\begin{aligned}
\mathfrak{p}_{2}(x) & =\prod_{i=1}^{m}\left[b_{i}-a^{i} \cdot x\right], \\
\mathfrak{p}_{1}(x) & =\prod_{F \in \mathcal{F}_{1}}\left[\left(b_{[F]_{1}}+b_{[F]_{2}}\right)-\left(a^{[F]_{1}}+a^{[F]_{2}}\right) \cdot x\right], \\
\mathfrak{p}_{0,(1,1,2)}(x) & =\prod_{v \in \mathcal{F}_{0}}\left[\left(b_{[v]_{1}}+b_{[v]_{2}}+2 b_{[v]_{3}}\right)-\left(a^{[v]_{1}}+a^{[v]_{2}}+2 a^{[v]_{3}}\right) \cdot x\right],
\end{aligned}
$$

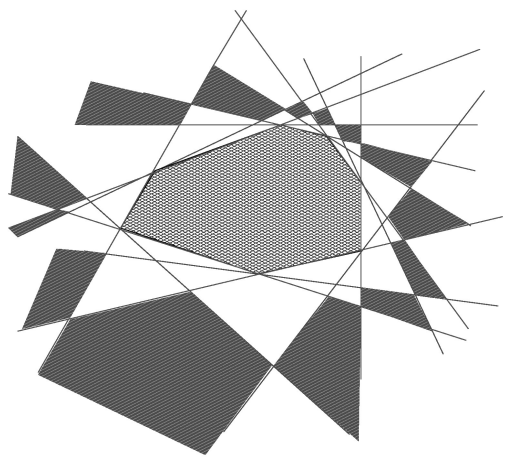

$\left\{x \in \mathbb{R}^{d}: \mathfrak{p}_{1}(x) \geq 0\right.$ and $\left.\mathfrak{p}_{0}(x) \geq 0\right\}$

Fig. 2 


$$
\begin{aligned}
\mathfrak{p}_{0,(1,2,1)}(x) & =\prod_{v \in \mathcal{F}_{0}}\left[\left(b_{[v]_{1}}+2 b_{[v]_{2}}+b_{[v]_{3}}\right)-\left(a^{[v]_{1}}+2 a^{[v]_{2}}+a^{[v]_{3}}\right) \cdot x\right], \\
\mathfrak{p}_{0,(2,1,1)}(x) & =\prod_{v \in \mathcal{F}_{0}}\left[\left(2 b_{[v]_{1}}+b_{[v]_{2}}+b_{[v]_{3}}\right)-\left(2 a^{[v]_{1}}+a^{[v]_{2}}+a^{[v]_{3}}\right) \cdot x\right], \\
\mathfrak{p}_{\bar{\varepsilon}}(x) & =\sum_{i=1}^{m} \frac{1}{m}\left[\frac{2 a^{i} \cdot x-b_{i}+h\left(-a^{i}\right)}{b_{i}+h\left(-a^{i}\right)}\right]^{2 p},
\end{aligned}
$$

where $\bar{\varepsilon}$ has to be chosen such that (3.13) is satisfied, and $p$ is given by Lemma 2.6. Let us consider a "real" three-dimensional polytope $P=\left\{x \in \mathbb{R}^{d}: A x \leq b\right\}$, with

$$
A=\left(\begin{array}{rrr}
0 & 3 & 2 \\
0 & -3 & 2 \\
2 & 0 & 3 \\
2 & 0 & -3 \\
3 & 2 & 0 \\
-3 & 2 & 0 \\
0 & -3 & -2 \\
0 & 3 & -2 \\
-2 & 0 & -3 \\
-2 & 0 & 3 \\
-3 & -2 & 0 \\
3 & -2 & 0
\end{array}\right), \quad b=\left(\begin{array}{l}
5 \\
6 \\
5 \\
4 \\
5 \\
5 \\
5 \\
6 \\
5 \\
4 \\
6
\end{array}\right)
$$

$P$ is a simple polytope with 12 facets, all of them pentagons, 30 edges, 20 vertices, and it may be described as a "skew" dodecahedron (see Fig. 3).

With respect to the facets we get the polynomial

$$
\begin{aligned}
\mathfrak{p}_{2}(x)= & \left(5-3 x_{2}-2 x_{3}\right)\left(6+3 x_{2}-2 x_{3}\right)\left(5-2 x_{1}-3 x_{3}\right)\left(4-2 x_{1}+3 x_{3}\right) \\
& \times\left(5-3 x_{1}-2 x_{2}\right)\left(5+3 x_{1}-2 x_{2}\right)\left(6+3 x_{2}+2 x_{3}\right)\left(5-3 x_{2}+2 x_{3}\right) \\
& \times\left(6+2 x_{1}+3 x_{3}\right)\left(5+2 x_{1}-3 x_{3}\right)\left(4+3 x_{1}+2 x_{2}\right)\left(6-3 x_{1}+2 x_{2}\right) .
\end{aligned}
$$

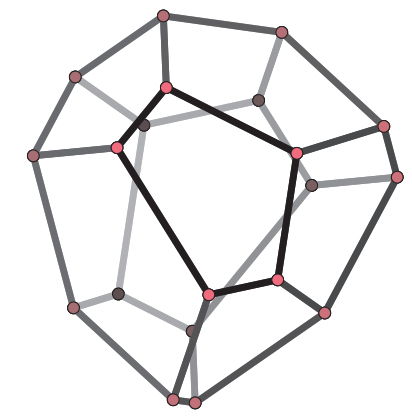

Fig. 3 (produced using polymake [GJ] and javaview [PKPE]) 
For the 30 edges we obtain

$$
\begin{aligned}
\mathfrak{p}_{1}(x)= & \left(10-2 x_{1}-3 x_{2}-5 x_{3}\right)\left(10+2 x_{1}-3 x_{2}-5 x_{3}\right)\left(10+3 x_{1}+-5 x_{2}-2 x_{3}\right) \\
& \times\left(10-6 x_{2}\right)\left(10-3 x_{1}-5 x_{2}-2 x_{3}\right)\left(11-2 x_{1}+3 x_{2}-5 x_{3}\right) \\
& \times\left(12-3 x_{1}+5 x_{2}-2 x_{3}\right)\left(12+6 x_{2}\right)\left(10+3 x_{1}+5 x_{2}-2 x_{3}\right) \\
& \times\left(11+2 x_{1}+3 x_{2}-5 x_{3}\right)\left(10-6 x_{3}\right)\left(11-5 x_{1}+2 x_{2}-3 x_{3}\right) \\
& \times\left(10-5 x_{1}+-2 x_{2}-3 x_{3}\right)\left(9-5 x_{1}-2 x_{2}+3 x_{3}\right)\left(10-5 x_{1}+2 x_{2}+3 x_{3}\right) \\
& \times\left(10-2 x_{1}+3 x_{2}+5 x_{3}\right)\left(10+6 x_{3}\right)\left(9-2 x_{1}-3 x_{2}+5 x_{3}\right) \\
& \times\left(10-3 x_{1}-5 x_{2}+2 x_{3}\right)\left(11-6 x_{1}\right)\left(10+5 x_{1}-2 x_{2}-3 x_{3}\right)\left(9+6 x_{1}\right) \\
& \times\left(11+5 x_{1}-2 x_{2}+3 x_{3}\right)\left(10+3 x_{1}-5 x_{2}+2 x_{3}\right)\left(12-3 x_{1}+5 x_{2}+2 x_{3}\right) \\
& \times\left(12+2 x_{1}+3 x_{2}+5 x_{3}\right)\left(10+3 x_{1}+5 x_{2}+2 x_{3}\right)\left(11+2 x_{1}+-3 x_{2}+5 x_{3}\right) \\
& \times\left(10+5 x_{1}+2 x_{2}+3 x_{3}\right)\left(9+5 x_{1}+2 x_{2}-3 x_{3}\right) .
\end{aligned}
$$

With respect to the 20 vertices we get three polynomials depending on the weights $w \in \mathcal{W}_{0}=\{(1,1,2),(1,2,1),(2,1,1)\}:$

$$
\begin{aligned}
\mathfrak{p}_{0,(1,1,2)}(x)= & \left(20-8 x_{1}-7 x_{2}-5 x_{3}\right)\left(20+2 x_{1}-3 x_{2}-11 x_{3}\right) \\
& \times\left(20-3 x_{1}+-11 x_{2}+2 x_{3}\right)\left(20+3 x_{1}-11 x_{2}+2 x_{3}\right) \\
& \times\left(20+7 x_{1}-5 x_{2}-8 x_{3}\right)\left(21+2 x_{1}+3 x_{2}-11 x_{3}\right) \\
& \times\left(23-8 x_{1}+7 x_{2}-5 x_{3}\right)\left(20+6 x_{1}+10 x_{2}\right)\left(24-6 x_{1}+10 x_{2}\right) \\
& \times\left(19+8 x_{1}+7 x_{2}+-5 x_{3}\right)\left(22-11 x_{1}+2 x_{2}-3 x_{3}\right) \\
& \times\left(19-5 x_{1}-8 x_{2}+7 x_{3}\right)\left(21+-11 x_{1}+2 x_{2}+3 x_{3}\right) \\
& \times\left(22+2 x_{1}+3 x_{2}+11 x_{3}\right)\left(22-8 x_{1}+7 x_{2}+5 x_{3}\right) \\
& \times\left(21+2 x_{1}-3 x_{2}+11 x_{3}\right)\left(22+7 x_{1}-5 x_{2}+8 x_{3}\right) \\
& \times\left(19+11 x_{1}+2 x_{2}+3 x_{3}\right)\left(18+11 x_{1}+2 x_{2}-3 x_{3}\right) \\
& \times\left(20+8 x_{1}+7 x_{2}+5 x_{3}\right), \\
\mathfrak{p}_{0,(1,2,1)}(x)= & \left(20-7 x_{1}-5 x_{2}-8 x_{3}\right)\left(20-2 x_{1}-3 x_{2}-11 x_{3}\right)\left(20-6 x_{1}+-10 x_{2}\right) \\
& \times\left(20+6 x_{1}-10 x_{2}\right)\left(20+8 x_{1}-7 x_{2}-5 x_{3}\right)\left(21-2 x_{1}+3 x_{2}-11 x_{3}\right) \\
& \times\left(22-7 x_{1}+5 x_{2}-8 x_{3}\right)\left(22+3 x_{1}+11 x_{2}+2 x_{3}\right) \\
& \times\left(24-3 x_{1}+11 x_{2}+2 x_{3}\right)\left(20+7 x_{1}+5 x_{2}+-8 x_{3}\right) \\
& \times\left(21-11 x_{1}-2 x_{2}-3 x_{3}\right)\left(19-8 x_{1}-7 x_{2}+5 x_{3}\right) \\
& \times\left(20+-11 x_{1}-2 x_{2}+3 x_{3}\right)\left(22+6 x_{2}+10 x_{3}\right)\left(22-5 x_{1}+8 x_{2}+7 x_{3}\right) \\
& \times\left(20-6 x_{2}+10 x_{3}\right)\left(21+5 x_{1}-8 x_{2}+7 x_{3}\right)\left(21+10 x_{1}+6 x_{3}\right) \\
& \times\left(19+10 x_{1}-6 x_{3}\right)\left(22+7 x_{1}+5 x_{2}+8 x_{3}\right),
\end{aligned}
$$




$$
\begin{aligned}
\mathfrak{p}_{0,(2,1,1)}(x)= & \left(20-5 x_{1}-8 x_{2}-7 x_{3}\right)\left(20-6 x_{2}-10 x_{3}\right)\left(20-3 x_{1}-11 x_{2}-2 x_{3}\right) \\
& \times\left(20+3 x_{1}-11 x_{2}-2 x_{3}\right)\left(20+5 x_{1}-8 x_{2}-7 x_{3}\right)\left(22+6 x_{2}-10 x_{3}\right) \\
& \times\left(23-5 x_{1}+8 x_{2}-7 x_{3}\right)\left(22+3 x_{1}+11 x_{2}-2 x_{3}\right)\left(24-3 x_{1}+11 x_{2}-2 x_{3}\right) \\
& \times\left(21+5 x_{1}+8 x_{2}-7 x_{3}\right)\left(21-10 x_{1}-6 x_{3}\right)\left(18-7 x_{1}-5 x_{2}+8 x_{3}\right) \\
& \times\left(19-10 x_{1}+6 x_{3}\right)\left(20-2 x_{1}+3 x_{2}+11 x_{3}\right)\left(20-7 x_{1}+5 x_{2}+8 x_{3}\right) \\
& \times\left(19-2 x_{1}-3 x_{2}+11 x_{3}\right)\left(21+8 x_{1}-7 x_{2}+5 x_{3}\right)\left(20+11 x_{1}-2 x_{2}+3 x_{3}\right) \\
& \times\left(19+11 x_{1}-2 x_{2}-3 x_{3}\right)\left(22+5 x_{1}+8 x_{2}+7 x_{3}\right) .
\end{aligned}
$$

Next we have to determine an $\bar{\varepsilon}$ as defined in (3.13). To this end we have estimated all the needed distances $\varepsilon_{k}$ (see (3.11)) by a rather ad hoc method and found

$$
\varepsilon_{2}>\frac{1}{10}, \quad \varepsilon_{1}>\frac{3}{50}, \quad \varepsilon_{0}>\frac{3}{100} .
$$

Hence we may set $\bar{\varepsilon}=\frac{3}{100}$ and since $\operatorname{diam}(P) \leq 4$ we may choose for the exponent $p$ of Lemma $2.6 p=332$. With these values we get from Lemma 2.6 the following polynomial $\mathfrak{p}_{\bar{\varepsilon}}(x)$ :

$$
\begin{aligned}
\mathfrak{p}_{\bar{\varepsilon}}(x)= & \frac{1}{12}\left[\frac{6 x_{2}+4 x_{3}+1}{11}\right]^{664}+\frac{1}{12}\left[\frac{-6 x_{2}+4 x_{3}-1}{11}\right]^{664}+\frac{1}{12}\left[\frac{4 x_{1}+6 x_{2}+1}{11}\right]^{664} \\
& +\frac{1}{12}\left[\frac{4 x_{1}-6 x_{3}+1}{9}\right]^{664}+\frac{1}{12}\left[\frac{6 x_{1}+4 x_{2}-1}{9}\right]^{664}+\frac{1}{12}\left[\frac{-6 x_{1}+4 x_{2}+1}{11}\right]^{664} \\
& +\frac{1}{12}\left[\frac{-6 x_{2}-4 x_{3}-1}{11}\right]^{664}+\frac{1}{12}\left[\frac{6 x_{2}-4 x_{3}+1}{11}\right]^{664} \\
& +\frac{1}{12}\left[\frac{-4 x_{1}-6 x_{2}-1}{11}\right]^{664}+\frac{1}{12}\left[\frac{-4 x_{1}+6 x_{3}-1}{9}\right]^{664} \\
& +\frac{1}{12}\left[\frac{-6 x_{1}-4 x_{2}+1}{9}\right]^{664}+\frac{1}{12}\left[\frac{6 x_{1}-4 x_{2}-1}{11}\right]^{664}
\end{aligned}
$$

\section{Outlook}

Why should anyone care about the representation of polyhedra by exponentially many polynomial inequalities, given that one knows that quadratically many suffice? Our answer is that the latter result is of pure existential nature, while we can construct such inequalities. We admit that the representations we found do not form an achievement of concrete practical value. That is why we did not state them in an algorithmic fashion. We see our paper just as a small step towards a development of real algebraic geometry in a constructive direction. There are a number of possible routes. We want to mention briefly what we are interested in and what might be achievable.

It would be nice to have efficient (in a sense that can be made precise) algorithms that provide, e.g., for polytopes $P$ given in the form of a $\mathcal{V}$ - or $\mathcal{H}$-representation, a $\mathcal{P}$ representation $P=\mathcal{P}\left(\mathfrak{p}_{1}, \ldots, \mathfrak{p}_{l}\right)$ with a number $l$ of polynomials that is polynomially 
bounded in the dimension of the polytope. It may also be useful to be able to construct a small number of "simple" polynomials $\mathfrak{p}_{1}, \ldots, \mathfrak{p}_{k}$ such that $\mathcal{P}\left(\mathfrak{p}_{1}, \ldots, \mathfrak{p}_{k}\right)$ approximates $P$ well. Of course, one can study similar problems concerning the representation of arbitrary semi-algebraic sets. For example, given a semi-algebraic set $\mathcal{S}$, can it be represented by a system of polynomials with total degree at most $k$, say? Can such a system be constructed efficiently? How well can $\mathcal{S}$ be approximated by polynomials of degree $k$ ? For polyhedra $P$ we know that there exists a representation by polynomials of total degree 1 , but what can we say about the minimum number of polynomials of degree $k$ representing $P$ ?

To indicate possible outcomes that may result from such a change of representation, we look at the very successful polyhedral approach to combinatorial optimization. The basic idea here is to represent combinatorial objects (such as the tours of a traveling salesman, the independent sets of a matroid, or the stable sets in a graph) as the vertices of a polytope. This way one arrives at an (implicit) $\mathcal{V}$-representation of classes of polytopes such as traveling salesman or stable set polytopes. If one can find complete or tight partial representations of polytopes of this type by linear equations and inequalities (i.e., $\mathcal{H}$-representations), linear programming (LP) techniques can be employed to solve the associated combinatorial optimization problem, see [GLS].

This approach provides general machinery to establish the polynomial time solvability of combinatorial problems theoretically. In particular, it is often employed to identify easy special cases of generally hard problems. One such example is the stable set problem that is $\mathcal{N} \mathcal{P}$-hard for general graphs but solvable in polynomial time for perfect (and other classes of) graphs, see Chapter 9 of [GLS].

The LP approach provides more. Even in the case where only partial $\mathcal{H}$-representations of the polyhedra associated with combinatorial problems are known, LP techniques (such as cutting planes and column generation) have resulted in very successful exact or approximate solution methods. One prime example for this methodology is the traveling salesman problem, see $[\mathrm{ABCC}]$ and the corresponding web page at http://www. math. princet on. edu/tsp/, which includes an annotated bibliography with remarks about the historical development of this area.

Progress of this type may also be possible via the "P -representation approach." We discuss this by means of the stable set problem.

Although complexity theory suggests that it is inconceivable that one can find an explicit $\mathcal{P}$-representation of all members of the class of stable set polytopes, i.e., the convex hull of all incidence vectors of stable sets, it might be possible to find, for every graph $G$, a "small" number $l(G)$ of not too "ugly" polynomials such that $\mathcal{P}\left(\mathfrak{p}_{1}, \ldots, \mathfrak{p}_{l(G)}\right)$ approximates the stable set polytope $\operatorname{STAB}(G)$ well, and such that $\mathcal{P}\left(\mathfrak{p}_{1}, \ldots, \mathfrak{p}_{l(G)}\right)$ equals $\operatorname{STAB}(G)$ for a special class of graphs.

It is also conceivable that, for such particular systems $\mathfrak{p}_{1}, \ldots, \mathfrak{p}_{l(G)}$ of polynomials, special nonlinear programming algorithms can be designed that solve optimization problems over $\mathcal{P}\left(\mathfrak{p}_{1}, \ldots, \mathfrak{p}_{l(G)}\right)$ efficient in practice or theory.

We do know, of course, that these indications of possible future results are mere speculation. Visions of this type, however, were the starting point of the results presented in this paper. We do hope that there will be progress in some of the directions mentioned. 


\section{Acknowledgments}

We thank Monika Ludwig and Günter M. Ziegler for helpful discussions, and Michael Joswig for pointing out a mistake in a previous version of Corollary 4.1 and for giving suggestions for improvements. We are grateful to the anonymous referees for their helpful comments.

\section{References}

[ABCC] D. Applegate, R. Bixby, V. Chvatal, and W. Cook, On the solution of traveling salesman problems, Doc. Math., J. DMV, Extra Vol. ICM Berlin 1998, III (1998), 645-656.

[ABR] C. Andradas, L. Bröcker, and J. M. Ruiz, Constructible Sets in Real Geometry, Springer-Verlag, Berlin, 1996.

[Ba] A. Barvinok, Approximating a norm by a polynomial, GAFA seminar notes (to appear), arXiv:math. FA/0105069.

[BCR] J. Bochnak, M. Coste, and M.-F. Roy, Real Algebraic Geometry, Springer-Verlag, New York, 1998.

[Be] A. Bernig, Constructions for the theorem of Bröcker and Scheiderer, Master's thesis, Universität Dortmund, 1998.

[BF] T. Bonnesen and W. Fenchel, Theorie der konvexen Körper, Berichtigter Reprint, 1974 edn., SpringerVerlag, Berlin, 1934.

[BG] A. Brieden and P. Gritzmann, On the inapproximability of polynomial-programming, the geometry of stable sets, and the power of relaxations, Preprint, TU München, 2001.

[BM] J. Burési and L. Mahé, Reducing inequalities with bounds, Math. Z. 227(2) (1998), 231-243.

[Br] L. Bröcker, On basic semialgebraic sets, Exposition. Math. 9 (1991), 289-334.

[BV] A. I. Barvinok and A. M. Vershik, Polynomial-time computable approximation of families of semialgebraic sets and combinatorial complexity, in Proceedings of the St. Petersburg Mathematical Society. Vol. I (O. A. Ladyzhenskaya and A. M. Vershik, eds.), American Mathematical Society, Providence, RI, 1993, pp. 1-17.

[CLO] D. Cox, J. Little, and D. O'Shea, Ideals, Varieties, and Algorithms, Springer-Verlag, New York, 1992.

[Fi] W. Firey, Approximating convex bodies by algebraic ones, Arch. Math. 25 (1974), 424-425.

[GJ] E. Gawrilow and M. Joswig, polymake 1.4.1, http://www.math.tu-berlin.de/diskregeom/ polymake, June 2001.

[GK] P. Gritzmann and V. Klee, Computational convexity, in Handbook of Discrete and Computational Geometry (J. E. Goodman and J. O’Rourke, eds.), Ser. Discrete Math. Appl., CRC Press, Boca Raton, FL, 1997, pp. 491-515.

[GLS] M. Grötschel, L. Lovász, and A. Schrijver, Geometric Algorithms and Combinatorial Optimization, Algorithms and Combinatorics, vol. 2, Springer-Verlag, Berlin, 1988.

[Ham] P. C. Hammer, Approximation of convex surfaces by algebraic surfaces, Mathematika 10 (1963), 64-71.

[Han] D. Handelman, Representing polynomials by positive linear functions on compact convex polyhedra, Pacific J. Math. 132(1) (1988), 35-62.

[La] J. B. Lasserre, Global optimization with polynomials and the problem of moments, SIAM J. Optim. 11(3) (2001), 796-817.

[Ma] L. Mahé, Une démonstration élémentaire du théoréme de Bröcker-Scheiderer, C. R. Acad. Sci. Paris Ser. I 309 (1989), 613-616.

[Mi] H. Minkowski, Volumen und Oberfläche, Math. Ann. 57 (1903), 447-495.

[MS] P. McMullen and G. C. Shephard, Convex Polytopes and the Upper Bound Conjecture, Cambridge University Press, Cambridge, 1971.

[MSW] J. Matoušek, M. Sharir, and E. Welzl, A subexponential bound for linear programming, Algorithmica 16(4-5) (1996), 498-516.

[PKPE] K. Polthier, S. Kadem-Al Charieh, E. Preuß, and U. Eitebuch, Javaview - 3D-geometry in web pages, http://www.javaview.de, February 2002. 
[PR] V. Powers and B. Reznick, A new bound for Pólya's theorem with applications to polynomials positive on polyhedra, J. Pure Appl. Algebra 164 (2001), 221-229.

[Sc] C. Scheiderer, Stability index of real varieties, Invent. Math. 97(3) (1989), 467-483.

[Se] R. Seidel, Convex hull computations, in Handbook of Discrete and Computational Geometry (J. E. Goodman and J. O'Rourke, eds.), Ser. Discrete Math. Appl., CRC Press, Boca Raton, FL, 1997, pp. 361-375.

[vH] G. vom Hofe, Beschreibung von ebenen konvexen $n$-Ecken durch höchstens drei algebraische Ungleichungen, Ph.D. thesis, Universität Dortmund, 1992.

[We] W. Weil, Einschachtelung konvexer Körper, Arch. Math. 26 (1975), 666-669.

[Zi] G. M. Ziegler, Lectures on Polytopes, Springer-Verlag, Berlin, 1995.

Received March 29, 2002, and in revised form October 24, 2002. Online publication March 19, 2003. 